\title{
Les écrits et les acteurs. Circulation des discours et empreinte des objets
}

Writing and acting. The propagation of discourse and marks left by written products.

\section{Sophie Pène}

\section{Q OpenEdition \\ Journals}

Édition électronique

URL : http://journals.openedition.org/edc/2497

DOI : 10.4000/edc. 2497

ISSN : 2101-0366

Éditeur

Université Lille-3

Édition imprimée

Date de publication : 1 janvier 1995

Pagination : $57-75$

ISSN : $1270-6841$

Référence électronique

Sophie Pène, "Les écrits et les acteurs. Circulation des discours et empreinte des objets », Études de communication [En ligne], 16 | 1995, mis en ligne le 22 juin 2011, consulté le 04 mai 2019. URL : http:// journals.openedition.org/edc/2497 ; DOI : 10.4000/edc.2497

Ce document a été généré automatiquement le 4 mai 2019.

(c) Tous droits réservés 


\title{
Les écrits et les acteurs. Circulation des discours et empreinte des objets
}

\author{
Writing and acting. The propagation of discourse and marks left by written \\ products.
}

Sophie Pène

1 Ecrit dit « professionnel » ou encore « ordinaire », « de travail », « au travail », « produits sur les lieux de travail », quelles que soient les périphrases, disgracieuses, mal commodes par lesquelles on les nomme, celles-ci expriment les embarras des utilisateurs que nous sommes face à une dénomination en creux et par défaut. Ces écrits sont loin de présenter une unité quelconque : les lieux de travail, les activités et les métiers auxquels ils sont associés, les préoccupations qui les motivent les différencient plus qu'ils ne les rapprochent. Ils ont en commun un seul trait, se dissocier de certains écrits à coup sûr en meilleure place dans la culture de l'écrit, les écrits des écrivains, ceux des journalistes et ceux aussi des enfants scolarisés, objets des études littéraires, de l'analyse de discours ou de la sociolinguistique à orientation didactique.

Cependant un nombre croissant de linguistes, de chercheurs en informationcommunication, de sociologues, d'anthropologues et d'historiens se tourne vers cet écrit qui, avant tout, n'est ni littéraire, ni journalistique, ni scolaire, ni didactique, ni fictionnel, ni poétique...

3 De $n i$ en $n i$, un besoin de bilan autour de quelques questions clés se fait sentir : l'originalité de cet objet d'étude est-elle une convention opportuniste ? Sommes nous avant tout animés par la satisfaction d'accumuler des observations de terrain étonnantes, fidèles à ce que l'ergonomie, la gestion et la sociologie disent de l'organisation du travail, de la construction des savoirs professionnels et des divisions sociales ? L'étude des écrits est-elle un témoignage parmi d'autres à propos des interrogations et des craintes des individus bousculés, menacés et épuisés par les changements socio-techniques ? Dans ce cas, l'écrit est simplement un observable parmi d'autres. Mais la matérialité de l'écrit suggère des approches attentives qui ne sont pas sans conséquence sur la connaissance de la langue et des formes du discours. Dans ce cas l'étude des écrits du travail répond à un 
besoin de connaissance et devrait devenir un champ de recherche à part entière, entraînant la maturation de méthodologies aujourd'hui embryonnaires ou un peu sauvages, et l'élaboration de concepts et de notions permettant les classements, les comparaisons et les transpositions.

\section{Écriture socialement située}

4 Le groupe d'étude Écrits écriture en contexte de travail (Gérico-CNAM) a entrepris la collecte et l'analyse de nombreuses formes d'écrits, par exemple les classiques comptes rendus d'intervention revus et corrigés à la mode de la qualité totale et du service rendu au client, les bilans d'entretien, les analyses de postes, les diverses façons d'« écrire le travail » et le savoir-faire, ainsi que les soucis que suscitent l'émergence ou le simple renforcement de tels écrits. D'autres questions débordent les terrains balisés par telle ou telle discipline : comment l'étude des écrits peut-elle apporter une contribution non anecdotique à l'étude du travail humain, prouver son opérativité pour observer l'établissement des savoirs de métier, par exemple la conception d'une procédure, par exemple la gestion d'un projet, par exemple la genèse de normes ? Ce qui est en question concerne aussi les attentes que le management place dans certains effets de l'écriture. La définition des tâches, la prescription et la motivation ont pris des chemins souvent dits « post-tayloriens ": l'organisation actuelle du travail remplacerait les bureaux des méthodes et la définition externe des étapes d'un processus de production par des méthodes d'auto-prescription. Celles-ci dépendent d'une description des pratiques réelles et d'une reconnaissance des bricolages individuels mises au jour et normalisées, entreprise qui suppose une enquête de chaque acteur sur son activité. L'intelligence engagée $^{1}$ ou l'implication subjective, comme on voudra l'appeler, est le moyen et la fin de cette démarche, dans laquelle les écrits jouent bien autre chose que leur rôle classique de trace ou de vecteur de communication. Ils reçoivent le matériau de ces comptes rendus d'expérience et sont la forme instituée de ces savoirs pratiques, qu'ils rendent comparables, à partir desquels ils entament des processus de normalisation. Le dialogue méconnu que le langage oral ou écrit entretient avec le travail est l'objet d'étude du Groupement de Recherche CNRS Langage et Travail. Comme en témoignent les travaux d'Anni Borzeix, de Josiane Boutet, de Daniel Faïta, de Bernard Gardin, de Michel Lacoste, de Jacques Girin et de Michèle Grosjean ${ }^{2}$, ce GDR analyse les productions langagières et leur circulation comme une source de l'action coopérative, de la catégorisation et de la séquentialisation des actes. Dans cette optique, parler et écrire sont des marques du lien social et aussi des bases du système cognitif.

5 C'est dire que le sujet est loin de s'épuiser. Au départ, des thématiques individuelles, des territoires de recherche singuliers, des héritages aussi. Michel Dabène ou encore Dominique Bourgain ont été des pionniers de la description de l'écrit « hors école », de sa pratique comme de ses représentations. Aujourd'hui, peut-être, un champ de recherche interdisciplinaire, à la croisée de plusieurs disciplines qui collaborent, convergent, s'opposent... en tout cas multiplient les points de vue. La variété des terrains n'est pas simplement le reflet des objets de recherche différents, mais aussi des histoires, des ambitions et des espoirs de recherche. Au premier chef, chez beaucoup d'entre nous, la volonté de faire connaître et reconnaître la littérature grise du travail autrement qu'en la saluant du mouvement de considération apitoyée qu'on accorde à un témoignage fade mais estimable. C'est sans sourire que nous défendons sa valeur, la puissance 
d'investissement et de manipulation qu'elle peut développer chez ses praticiens, les astuces que la domination des obstacles, langagiers ou politiques, fait inventer. Bien sûr nous ne crions pas au chef-d'oeuvre inconnu, même si dans le présent numéro, David Charrasse rapporte de beaux exemples de créativité stylistique, même si Pierre Delcambre (in Dumont et Revuz, di., 1994) s'attarde sur les « jugements de beauté » et les émotions qui s'expriment dans des ateliers d'écriture sur des sites professionnels.

\section{La force de l'écrit en train de se faire}

Peut-être faut-il prendre le temps d'évoquer la façon dont s'impose l'attention portée à l'écriture « en train de se faire ", manie partagée dès qu'il s'agit d'observer, de collecter, de décrire des dispositifs et des dispositions d'écriture. A n'en pas douter l'activité de formation est une racine vive. Tous ceux qui ont assisté des groupes d'étudiants et d'adultes dans la gestation d'un écrit savent bien la pénibilité très particulière que génère l'exercice. Exaltation et omnipotence au moment de la description du projet, ou au contraire intentions prudentes, modelées par la dénégation « je ne sais pas écrire, j'aimerais bien ... mais je ne sais pas écrire, je n'ai pas le temps, j'ai déjà d'autres efforts à faire », ou aussi « je ne vois pas à quoi ça peut me servir ». Et même quand l'animateur est tout prêt à adhérer aux objections, presque à s'excuser de ses exigences, à négocier des allégements acceptables, il est surpris de l'obstination avec laquelle l'objet écrit est investi.

7 Je pense également à la bonne volonté effrayante, incongrue, avec laquelle certains étudiants qu'on ne saurait soupçonner de graphomanie, raniment impétueusement des consignes fort raison-nables, assenant cinquante pages quand on en demande vingt, attendant un retour, un commentaire, impossible à donner avec justice, toujours insatisfaisant, semble-t-il, face à un engagement mystérieux, intime, celui d'une mise en mots jamais facile et jamais indifférente, même si une promesse d'habileté est donnée, même si une menace de désinvolture est brandie, même quand la contrainte préside et écrase l'envie d'écrire. Les écrits lient les étudiants à leurs objets de pensée. Ils les lient aussi aux institutions qui les exigent, aux personnes des enseignants qui « pilotent ». Un léger syndrome de Stockholm n'est sans doute pas étranger au plaisir immodeste dont jouissent ceux qui ont clos leur projet. Jamais on ne vit sujet plus intéressant, plus rondement mené, meilleurs conseils de bibliographie, ... et l'étudiant de feuilleter le document crissant, de promettre une suite, un complément pour une partie pas assez développée. C'est une concentration physique toute particulière que de relire un texte que l'on vient d'écrire, demi-propriété, demi-étrangeté, à la fois un bien, un élément du patrimoine, et un prolongement de soi, émanant de l'identité, du sentiment de la personnalité. Et c'est avec la même euphorie contenue que l'auteur achève la réalisation de l'objet de communication : tirages et retirages, dernières et dernières et encore dernières mises en page, photocopies, reliure, dépôt. Tout est bon pour cultiver une relation physique à l'objet écrit. Engagement corporel, engagement intellectuel, le travail d'écriture, suivi sur une longue durée, semble modifier substantiellement certains aspects de l'image que le scripteur a de ses compétences à planifier, à réaliser et à réviser un tel objet langagier ${ }^{3}$.

8 Pour beaucoup de " chercheurs de l'écrit ", l'expérience d'enseignement, en particulier auprès d'adultes, a précédé la fréquentation de l'écrit au travail, incitant sans doute à accorder une valeur forte aux marques de l'engagement des sujets. Il ne faut pas oublier 
non plus le rôle qu'ont joué les ateliers d'écriture pour les professeurs de lettres, participants ou animateurs. En créant les conditions favorables à l'expression et au plaisir d'une écriture souvent oralisée, ils restaurent une circulation de textes ordinairement renfermés dans l'intime, associant à tous les textes un pouvoir d'émotion esthétique, en interaction, presque sans critère de mérite. Au pôle opposé, celui du non littéraire, du non volontaire, du non gratuit, les salariés en formation interne étonnent par la richesse de leurs discours sur l'écrit, par la conscience de son efficience sociale de reproduction, d'exclusion ou de distinction. Des récits lucides affirment que l'écrit rend visible l'action professionnelle pour une hiérarchie distante, qui ordonne l'information, oriente les décisions, assure des avancements. Communication différée, in absentia, et inégale, l'écrit est pourtant un objet de fascination, de jouissance et de conquête, bien au-delà de la fonctionnalité, parce qu'il est langage : un langage particulier qui met en jeu le mécanisme doublement réflexif ${ }^{4}$, postulé par Vygotsky. Mais aussi parce qu'il découvre une marge pour l'imaginaire et le jeu, qui peut créer un territoire absolument individuel.

\section{Objets langagiers, objets du monde}

9 En fait, quelle que soit la trajectoire des chercheurs qui s'intéressent à l'écrit, tous ont un attachement fort, personnel à l'objet. Un objet qui, comme masse, a quelque chose d'écrasant. Montée quantitative, diversité qualitative, corrélation entre changement organisationnel et apparition de nouvelles formes d'écrits, dans la gestion des ressources humaines, dans la démarche qualité et la certification ISO $9000^{5}$, dans la formalisation de procédures, dans la relation avec les clients des entreprises. Cette diversité dégage-t-elle une intelligibilité, une comparabilité, au-delà des singularités? L'engouement pour l'inscription des savoirs, pour l'analyse de la pratique professionnelle, des tâches, des postes, des métiers, passe par des dispositifs d'écriture plus ou moins élaborés ; ces pratiques ont sans doute une signification globale pour notre société post-industrielle. Comment trouver et ordonner les marques linguistiques ${ }^{6}$, discursives et graphiques ${ }^{7}$ qui aideraient à caractériser ces formats d'écrits et à reconnaître la transtextualité propre à ces ensembles de textes?

10 C'est naturellement sur des objets en relation étroite avec l'activité professionnelle que les études de terrain se conduisent, avec des bizarreries constitutives qui imposent des inventions, des ruses et des compromis. Les écrits, les procès d'écriture, la circulation des discours dans les univers professionnels n'ont pas le contour pur de certains objets d'étude. C'est une recherche sans sécurité dont la progression dépend de la connaissance de l'activité et des acteurs, imprégnant la constitution des corpus d'une dimension anthropologique. On est loin du linguiste qui produit lui-même un énoncé pour vérifier la possibilité d'une construction syntaxique. On est loin de l'analyste de discours, qui étudie un lexique, une forme propositionnelle, ou une thématique dans un corpus d'écrits. On est loin aussi du spécialiste de génétique textuelle qui cherche les règles implicites de réécriture des versions d'un écrit littéraire à partir d'un corpus de variantes. C'est pourtant bien d'objets langagiers que nombre d'entre nous s'occupent. « Objets langagiers " s'entend dans son sens plein : objets, parce que la matérialité des mots et du discours importe. Les séquences du discours, la disposition dans la page, le choix du support sont pris en compte. Mais objets aussi comme objets du monde ayant une substance, une épaisseur, un poids ; objets de transaction, d'interaction, d'archivage, expression de rituels de travail, repères des divisions des territoires et des pouvoirs. 
11 Tout en se séparant par tous ses aspects anthropologiques et sociologiques de la linguistique au sens strict, c'est cependant à partir de théories linguistiques, ou de résultats de l'analyse génétique, par exemple, qu'une linguistique de l'écrit se développe du côté des écrits ordinaires. La linguistique de l'énonciation guide l'étude des marques de la prise en charge du discours par un sujet, aide à repérer l'organisation de tours d'écriture, les énonciations multiples, "hétérogènes ", " plurielles » ou ces sortes de strates d'énonciation que déposent les en-tête, les logos, les hiérarchies, les noms propres apposés en vedette, les numéros de téléphones offerts en recours, les signatures manuscrites, les tampons, les « pour ordre » ou les griffes. Le dialogisme de Bakhtine structure l'idée de circulation des discours, par les reformulations, l'installation des formes, la genèse des normes. Et c'est bien pour étendre la description des emplois et des formes de la langue que nous donnons leurs lettres de noblesse aux tours d'écriture, partie des tours de métier, aux formes mal connues des écrits issus du travail, écrits de la technique, écrits de la gestion, écrits des professions : à l'instar des ouvriers, des infirmières, des agents administratifs, des agents de maintenance, des personnels du secteur sanitaire et social, chacun d'entre nous est un locuteur, un scripteur qui puise dans son métier des habitudes et des formats d'écriture, des genres rédactionnels qui sont aussi des objets du monde, occupant un espace, susceptibles d'être mesurés, qualitativement et quantitativement.

\section{Des écrits pris dans le cours d'action}

12 Là sont l'incertitude, la difficulté et la charge du projet. Le linguiste de terrain s'occupe des pratiques langagières. Rien de mystérieux : le langage articulé se différencie du silence et du cri. Le langage écrit se reconnaît à la matérialité des mots, du support, du tracé. La constitution de corpus semble simple. Ne s'agit-il pas de recueillir une matière à analyser ? Cependant l'écrit du travail n'est pas un écrit autarcique, autonomisable de son environnement. S’il rend compte du réel en le modélisant par une procédure, en le miniaturisant par un dessin ou par une photo, en le décrivant par un compte rendu ou un rapport, c'est pour ainsi dire en plus et par accident. Ce n'est pas sa vocation première, ni sa vocation unique.

13 Pour prendre un exemple constrastif, le cas du compte rendu littéraire d'une action telle qu'un voyage montre un rapport beaucoup mieux dissocié entre l'action ou l'événement et leur trace écrite. Le Journal de René Caillié ${ }^{8}$ rend compte de son périple vers Tombouctou. Attentif aux aspects géographiques, ethnographiques, linguistiques des pays qu'il traverse, René Caillié accumule portraits, plans de mosquée, notes sur la faune et la flore. Narrant, décrivant, Le Journal est un récit centré sur la restitution pour soi et pour autrui, en l'occurrence la Société de géographie et le public, d'événements, de faits, d'impressions. Un auteur, une expérience vécue, sa mise en mots : le projet, si opaque soit-il à un plan littéraire, se représente avec cohérence et relative simplicité. La gestion de l'expérience par le genre littéraire « journal de voyage » est un modèle culturellement identifié.

14 Tout autres sont la production et la réception des écrits qui nous occupent, et aussi leurs statuts dans l'imaginaire de l'observateur. Au lieu de cet écrit non fictionnel en cohésion avec une histoire et une subjectivité, l'écrit du travail est donné dans une espèce de chaos déstabilisant pour le linguiste. Ne serait-ce que parce qu'il faut comprendre l'objet technique, le type de raisonnement, de décision, d'environnement réglementaire, 
juridique, organisationnel, stratégique que le texte porte en filigrane. Certes un compte rendu de réunion est au même titre que tout écrit un aménagement de divers procédés de description et de narration. Mais la projection (l'intrusion ? la figuration?) du réel dans le texte n'a pas la même homogénéité que dans des écrits qu'une personne rédige, parce qu'elle en a envie ou parce qu'elle se le doit. L'écrit fait partie du travail, il n'en est pas une reformulation, il est pris dans le travail. Écrire au travail n'est donc pas un retrait temporaire "hors de l'action ", comparable à la mise hors d'eau d'un bateau. Peu de méditation et rien d'érémitique dans l'écrit du travail, qui est un écrit du contact interpersonnel et intersémiotique, un écrit de la mise en tension entre monde de la personne et organisation, entre subjectivité et point de vue extérieur.

\section{Les navettes sémiotiques entre écrit, oral, iconique}

Fait passionnant pour le linguiste, cet écrit illustre à merveille les situations de chevauchement entre sémiotiques que la sociolinguistique de terrain pressent, défend et décrit. D'une linguistique de l'oral ou d'une linguistique de l'écrit, on passe à une linguistique de l'écrit et de l'oral, de l'écrit dans l'oral et de l'oral dans l'écrit. Les formes écrites sont en effet toujours insérées dans de multiples rebonds : c'est après une réunion qu'on rédige une note, ensuite diffusée. Cette note peut se retrouver dans un même bureau affichée, archivée dans un dossier, glissée dans un autre en cours de traitement. Des fragments seront cités, soit dans un autre texte, soit dans une conversation, soit dans un exposé de travail. Autre exemple de la circulation des discours : un responsable d'étude rend compte d'un projet dans un rapport intermédiaire. Pour le diffuser, il compose une synthèse initiale. Pour la réunion de travail, cette synthèse donnera la matière et la stratégie de présentation, et le hiérarchique la reprendra à son tour dans le compte rendu oral et écrit qu'il fera en réunion de service. Ces situations de communication successives et interdépendantes, impliquant des chaînes de locuteurs et de scripteurs, ne déterminent pas simplement des effets de copiage, de citation, de répétition. Le traitement sémiotique proprement dit est affecté par la variété des formes et des moments des échanges.

En fonction de chacun des événements évoqués, les énonciateurs ordonnent, composent, s'efforcent d'ajuster, dans l'interaction anticipée ou immédiate, une mise en mots chaque fois neuve. Les analyses et les préconisations prennent des accents différents en fonction des préoccupations de l'auditoire. Ce ne sont pas simplement la cadence d'un exposé, son niveau de complexité ou l'alternance " français parlé " "français écrit 》 qui interviennent. C'est la façon même dont la construction du sens et le réel découpé, perçu et rapporté, évoluent, laissant à chaque étape des strates dans le discours ensuite réimporté.

\section{La cohésion de l'action : inventer, communiquer, vérifier}

De tels processus s'observent à petite échelle, un thème de travail précis qu'on suit sur quelques semaines. Des dispositifs plus lourds pourraient en rapporter des marques, dans une même entreprise, à propos d'intervalles de plusieurs années. Il est facile d'en être conscient au vu des thématiques développées managérialement : la culture d'entreprise, 
les contrats d'objectifs, l'idée de « changement » sont des fils évidents dans l'étude de la presse d'entreprise ou des circulaires. Plus subtilement et par d'autres méthodologies que l'analyse de discours, on pourrait s'interroger sur le succès d'un figement de langue, d'un énoncé qui " prend ", d'une mise en page séduisante qui fait école, d'un formulaire innovant, inventé, qui devient modèle, sans aucune intervention hiérarchique, et stabilise, normalise des solutions individuelles bricolées par différents individus, hors intervention hiérarchique.

Étudier les écrits de travail, c'est prendre en compte la simultanéité des interactions humaines, c'est suivre la successivité d'un procès, c'est aussi retrouver les transactions entre individus : qui prend en charge, dans le discours, telle décision, à quel moment, sous quelle forme ? Par la simple apposition d'un $O K$ assorti d'initiales en marge d'un programme d'étude? Par la signature d'un très officiel ordre de mission? Les réécritures successives et la prise en charge des morceaux de discours par un individu mandaté en " porte-écriture ", à l'instar du " porte-parole » décrit par B. Gardin, sont des éléments de la distribution du pouvoir/devoir d'écrire, inséparables de la forme des écrits.

Il n'est pas de discours en entreprise qui ne soit d'une façon ou d'une autre " déjà là ", illustrant dans un univers restreint, délimité, la théorie du dialogisme. Celle-ci se prête dans ce cadre à des exploitations très directes, telles que le repérage des reprises et des reformulations, l'étude du destin des formules. On comprend l'intérêt pour la linguistique de terrain de se plonger dans ces domaines où une ambiance de chancellerie et une obsession de la trace ont poussé à l'extrême l'art de l'archive et de la diffusion, renforçant simultanément l'activité de flux parallèles plus directs, visibles ou non, l'art du message, du post-it, de la prise de notes. Il est facile, presque facile, de voir à l'oeuvre, à partir de mêmes matières, différents traitements. Je viens d'évoquer l'imbrication de différentes situations d'interactions.

L'étude des feuillets d'un dossier est une ressource qui permet une autre approche, l'étude des discours hétérogènes qui coexistent en synchronie dans un " dossier " et maintiennent à flots différents plans de l'activité, ceux des choses à faire, à dire, à penser. Le dossier est l'unité de classement d'une "affaire ", d'un thème de travail régulier ou prolongé. Il a, d'un point de vue historique, une dimension d'archive, de mémoire ou de preuve du travail. Considéré comme un outil de gestion cognitive, il rassemble les marques du bilan et du projet, du certain, de l'hypothétique et du prédit, alliées à autant de discours hétérogènes les uns aux autres : un compte rendu daté, tamponné, signé, adressé, aura un tout autre statut que le feuillet manuscrit arraché d'un bloc sur lequel un numéro de téléphone évoque une personne à rappeler, tandis que des listes de groupes de mots soulignés en rouge ou en bleu, des biffures, des ajouts marqueront le monologue intérieur, la recherche d'idées, la démarche de planification. Des notes prises en entretien, un message d'une autre main indiquant une confirmation de rendez-vous, des doubles de commandes, des devis inaboutis, des échanges de courriers originaux ou dupliqués, annotés à l'intention d'un hiérarchique, la photocopie d'un extrait d'un décret d'application d'une loi, avec des passages surlignés à la main, sont autant d'exemples d'une subtilité de discours inachevés montrant l'activité sémiotique " en train de s'élaborer ", nécessaire à la poursuite de l'action coopérative complexe. 


\section{Le terrain : ouverture, imprégnation et conclusion}

21 Il y a donc un gisement d'études infini, offert aux chercheurs. D'autant que ce que j'évoque ainsi n'est qu'un des multiples parcours possibles. Chacun développe, en fonction des secteurs d'activité qu'il fréquente, des préoccupations et des méthodes adaptées à un site industriel ou tertiaire donné. Avec toutefois des soucis partagés, par exemple l'attention portée à l'incidence des pratiques langagières sur l'activité, ou la focalisation de l'intérêt sur la personne au travail, ou encore la recherche des équilibres entre les marques de la normalisation des actes du travail et le maintien d'un territoire d'innovation et de liberté.

Si le rapport au terrain est si important, c'est entre autres choses qu'il pose la question de la relation entre écriture et professionnalisation. Lier, comme Olivier Chantraine ou Pierre Delcambre (dans le numéro 13 d'Etudes de Communication par exemple), analyse des écrits et métier ou secteur d'activité oriente la recherche; ils observent que les métiers du secteur sanitaire et social reflètent une division du travail étrange entre " celui qui écrit » et « celui qui n'écrit pas ». D'autant que ce qu'eux-mêmes, d'expérience, savaient de ces pratiques ne correspondait pas à ce qu'en disaient les acteurs. D'où l'envie d'observer la réalité du partage de l'écrit, projet orientant la recherche et le type de résultats. Les routines du métiers, les rituels de la communauté sont des éléments qui s'étudient dans la continuité d'une histoire professionnelle ou, plus simplement, dans la séquentialité des travaux d'une journée : à quel moment se retourne-t-on vers l'écriture ? Après avoir fait quoi, et avant quoi ? Pour noter quoi ? Pour passer à une phase de réflexion, de retrait, ou au contraire pour formuler des consignes, faire de l'administration? Sous ces différents angles, écriture et édification de la compétence professionnelle développent des liens privilégiés.

Autre exemple, l'entrée par un type d'écrits : Françoise Rouard constitue des corpus d'écrits de maintenance d'équipements informatiques. Le compte rendu de l'action, la gestion, en discours, de l'échec ou de la réussite de la réparation, et des risques encourus face à la hiérarchie, sont l'occasion de montrer l'acteur se débattant dans la forme contrainte du compte rendu, en déjouant la neutralité pour se couvrir et mettre en scène une action optimisée. L'écrit est un complément indispensable de l'activité, il la justifie, la met en scène, la recompose.

Les démarches sont variées, mais toutes difficiles. Au plan de la démarche de terrain d'abord, au plan d'une certaine impureté d'objet d'autre part, au plan du statut et de la pertinence des résultats obtenus. L'ouverture de terrain n'a rien de spontané. Il est sans doute assez facile d'observer les pratiques langagières d'enfants au sein d'une classe. On peut prendre - pourquoi pas ? - son propre univers d'enseignant comme objet. On peut aussi s'appuyer sur les amitiés de métier. De toute façon rendre légitime son intervention ne posera pas de problème. Dans un site de travail, c'est différent, pour plusieurs raisons. L'entreprise d'aujourd'hui se protège. Elle protège le secret de sa vie interne, comme elle se protège de concurrents potentiels ou d'indiscrétions nuisibles. Elle se protège aussi parce qu'elle se vit comme lieu privé, ayant d'ailleurs, le droit le montre, un statut de personnalité morale qui la rapproche juridiquement de la famille plus que de l'État. Elle se protège enfin parce qu'elle est animée par des personnes, qui pratiquent fort normalement un goût du retrait, n'ont pas nécessairement l'autonomie suffisante pour accepter un comparse observateur dans leur bureau, et qui, surtout, réductions de 
personnel et évolutions continues obligent, sont très souvent accablées de travail, pressées, inquiètes. Par ailleurs, l'entreprise est, d'une façon un peu contradictoire, intéressée par un regard extérieur. Mais elle entend rester maitresse de ce qu'elle donne à voir. Là encore, le point d'entrée a une incidence : l'ouverture de terrain qui s'opère à un niveau de direction ne permet pas le même projet que celle qui démarre à partir d'un service opérationnel.

Quelle que soit la démarche qui se dégage, les histoires de terrain ont en commun d'être longues et fragiles, mais aussi sérieuses. Sérieuses, au sens où il n'est pas possible de voguer d'un métier à l'autre, car il y a des difficultés de compréhension pour le chercheur, des apprentissages longs des pratiques professionnelles locales. Il ne s'agit même pas d'aborder vraiment les savoirs techniques. Simplement pour avoir une espèce de carte mentale des territoires, des métiers, reconnaître d'un service à l'autre les mêmes programmes de travail présentés à un autre moment du développement, s'habituer à des jargons juste ce qu'il faut pour suivre le discours, cela demande du temps, une inscription longue, une familiarité qui permette une présence proche, sans faire de l'observateur une tache dans le tableau.

La difficulté de méthode, voire de théorie, se profile ; est-on encore dans une démarche de description des pratiques et des objets de langage ? Si pour lire, classer, analyser un énoncé, on est obligé de le remettre dans un contexte qui s'étend bien au-delà du texte et comprend sans limite l'environnement de travail, à coup sûr l'observateur emprunte à l'acteur certains positionnements, et la linguistique perd ses frontières langagières pour glisser vers une anthropologie difficile à faire reconnaître par une quelconque communauté scientifique. Quelle que soit la discipline d'origine, l'objet hétérogène qu'est le «travail humain et ses marques » impose de toute façon une extension des territoires habituels et un sentiment de fragilité des études : pour maintenir une comparabilité, un échantillonnage, il faudrait arriver à travailler sur des corpus bien nets, par exemple un ensemble de notes de services. Mais ce découpage a priori élimine toute chance d'une observation du langage en situation et banalise la démarche de terrain, pour en faire simplement une sorte de thématique accidentelle : avant-hier la presse, hier l'univers scolaire, aujourd'hui l'univers de travail. Et ce qu'un tel découpage empêche, c'est d'aller vers l'écrit associé au cours d'action.

Or l'étude des écrits a un intérêt majeur pour approcher le travail. Quelques indications : chacun sait l'importance de la préfabrication des écrits (formulaires, lettres type, préimprimés). On en tire des conclusions spontanées sur une incompétence rédactionnelle des acteurs. Si ces " prêt-à-écrire » (comme les appelle S. Branca-Rosoff à propos de la correspondance des soldats de 1914) expriment un souci d'automatiser, d'accélérer et de fiabiliser des activités rédactionnelles, il se développe cependant, simultanément, une pression antagoniste qui tend à pousser le salarié à prendre des risques d'auteur, écho des risques d'acteur : rédiger des lettres "personnalisées ", interpréter, évaluer la gravité d'un contentieux, décider de l'attribution d'un prêt bancaire en transgressant les procédures, engager sa signature sur la « qualité totale » d'un produit. Cette contrainte de risque, qui coexiste avec la standardisation des écrits, très visible pour un analyste attentif à la matière langagière, à l'étude du support, de la mise en texte et des modes de traçage, attire l'attention sur l'importance de rechercher les significations managériales des différents régimes d'écriture proposés aux salariés. Certains établissements instituent des chartes graphiques, respectées ou pas. Les bibliothèques de lettres types peuvent se constituer par collation de lettres diverses ou être rédigées de bout en bout par un 
cabinet de consultants. Pour peu que l'on regarde comment s'établissent les formes d'écrit, on saisit l'intérêt d'une économie de l'utilisation des formats. Catégoriser, classer, ordonner, rassembler pour autrui, c'est exercer un pouvoir, qui revient à solliciter certains discours et à en interdire d'autres. De la même façon, une technologie d'écriture, le fax ou l'informatique sur réseau ou non, induit une certaine façon " conforme » de rencontrer autrui, de partager ou non ses savoirs et ses informations. Commander dans un secrétariat certains objets de papeterie, des post-it, des blocs ou des cahiers détermine la gestion des supports et donc des discours, la mise en espace influant sur la mise en mots. Une histoire et un observatoire des techniques et de la sociabilité du travail sont dépendants de la capacité à recenser et analyser une culture de l'écrit.

Cela concerne aussi l'étude de l'établissement des savoirs. L'extraction de savoir-faire, et leur mise en mots, la sélection par ce biais entre des pratiques concurrentes rendues évaluables montrent comment l'écrit sert une certaine routinisation des savoir-faire, un examen de l'activité et contribue à la genèse de normes.

Enfin, les pratiques d'écrit sont aussi des pratiques sociales, des comportements de travail. L'ordinateur du bureau contient la correspondance personnelle, le courrier administratif du foyer. Les fichiers ont souvent des titres qui sont des prénoms, montrant la prégnance de la relation dans la catégorisation de documents. L'ordinateur favorise-t-il des chantiers d'écriture simultanés, gérés en superposition et en alternance, un quart d'heure pour un courrier urgent, quelques minutes de jeu ? Et puis beaucoup de salariés équipés d'ordinateurs ou de photocopieuses sont des ressources pour des associations dont ils sont membres. Des secrétaires décrivent des cadres " multimétiers ", intégrant à leur fonction essentielle des responsabilités associatives, des activités d'enseignement ou la participation à des conseils d'administration, dont elles sont amenées à assurer bon gré mal gré le support écrit. De ces comportements professionnels, l'écrit témoigne, offrant des chemins méconnus à l'observation.

En situation inconfortable au regard des pratiques de recherche classique, les observateurs de l'écrit du travail sont à une étape délicate. Des comptes rendus d'enquête s'accumulent, sans qu'à proprement parler une production théorique s'effectue. Le plaisir des coopérations d'équipe pallie sans doute la modestie d'ambitions notionnelles. Il y a pourtant un doute régulier : que faire de ces études si fortement rattachées à un site, comment en exploiter les résultats, comment arriver à des corpus significatifs, mais non simplifiés, qui permettent cependant des exploitations comparatives?

\section{Décrire l'écrit : quel gain pour les acteurs du travail ?}

31 Autre question d'importance : s'il est vrai que beaucoup des propos sur l'écrit de travail mettent en avant la prise en compte des rédacteurs, quel gain trouvent ces derniers à ces études ? Quel retour en parvient ? La valorisation de la pratique d'écriture professionnelle a des effets. Pour les salariés eux-mêmes, elle converge avec leur propre sentiment. En travaillant avec des groupes professionnels, de secrétaires ou d'agents administratifs, on met au jour, pour eux-mêmes et pour leur hiérarchie, la complexité de leurs tâches. Ainsi la pratique est respectée et l'offre de formation aux " techniques d'expression » peut être discutée et affinée. Les stéréotypes de la " phrase courte », de l'« écrire pour être lu ", ou " pour agir " s'assouplissent et s'imprègnent de conditions de lisibilité spécifiques au métier et à la situation. De la même façon, les interventions managériales sur les correspondances clients ou les journaux d'entreprise peuvent perdre 
leur caractère carré, à la lumière des problématiques défendues. Le point de vue des acteurs est pris en compte et c'est à partir de lui que le discours écrit est organisé, par le biais d'ateliers ou de groupes d'échanges des pratiques. Du moins est-ce dans cette direction que les observateurs engagés orientent d'éventuelles préconisations. Car c'est une particularité de ces interventions, justement soulignée par D. Charrasse : l'observateur qui intervient sur un site pour comprendre les pratiques d'écriture est toujours expert à un titre ou un autre. Formateur, chercheur, consultant, il a pour ses interlocuteurs une compétence supposée et une expérience pratique. C'est un genre de " militant » de l'écriture, ce qui le rend intéressé à un type de résultat, la réappropriation par les acteurs d'un statut ou d'un sentiment d'auteur.

\section{BIBLIOGRAPHIE}

Anis, J., (1983), « Le signifiant graphique », Langue française 59, Larousse.

Bertin, J., (1987), « La représentation graphique », Encyclopaedia Universalis, volume 7.

Bourgain, D., (1988), Discours sur l'écrit, analyse des représentations sociales de l'écrit en milieu professionnel, Thèse d'Etat, Université de Besançon.

Dabene, M., (1987), L'adulte et l'écriture, contribution à une didactique de l'écrit, Bruxelles, de Boeck.

Boutet, J., (dir.pub.), (1995), Paroles au travail, L'Harmattan.

Boutet, J., (1994), Construire le sens, Bern, Peter Lang.

Boutet, J., Gardin, B., Lacoste, M., (1995), « Discours en situation de travail », Langages n 114, mars 1995.

Branca-Rosoff, S., (1990), « Conventions d'écriture dans la correspondance des soldats », Mots $\mathrm{n}^{\circ}$ 24.

Communications, (1994), «L'écriture des sciences de l'homme », Le Seuil, nº 58.

Dejours, C. et Molinier, P., (1994), « Le travail comme énigme », Sociologie du Travail XXXVI Hors série/1994, p. 35-44.

Dumont, M. et Revuz, C., (dir. pub.), (1994), « Ecriture, travail, formation, Éducation Permanente n - 120.

Etudes de Communication, (1992), «Pratiques d'écriture et champs professionnels (2) », nº 13.

Fraenkel, B., (dir. pub.), (1993), Illettrismes, Centre Georges Pompidou, BPI, collection Études et Recherche.

Gardin, B. et Richard-Zappella, J., (1987), « Les porte-parole en question », La Pensée, 254, p. 87-100.

Jarry, I., (1992), René Caillié, J.-Cl. Lattès.

Lacoste, M., (1994), « Langage et travail : quelques perspectives », Sociologie du Travail XXXVI Hors série/1994, p. 45-56. 
Merle, V., (1993), « Points de vue socio-économiques sur l'illettrisme », in FRAENKEL, B. (dir. pub.), Illettrismes, Centre Georges Pompidou, BPI, collection Études et Recherche.

Pavillard, S., (1994), «Le récit de voyage, écriture et réécritures », Les Cahiers de l'I.U.T., mars 1994.

Schneuwly, B., (1988), « La conception vygotskienne du langage écrit », Études de linguistique appliquée, $\mathrm{n}^{\circ} 73$, p. 108-117.

Serieyx, H., (1994), L'effet Gulliver, Calmann-Lévy.

Vygotsky, L. S., (1934/1985), Pensée et langage, Paris, Éditions Sociales.

Zarifian, P., (1993), « Travail industriel, socialisation et libertés », Futur antérieur, « Paradigmes du travail », L'Harmattan.

\section{NOTES}

1. J'emprunte l'expression à $\mathrm{H}$. Sérieyx : «A partir du moment où les dirigeants demandent à leurs salariés de recevoir, de comprendre et d'émettre de l'information, ils font appel à l'intelligence engagée des personnes ». Sérieyx, (1994). H. Sérieyx fait porter ses propos sur les initiatives demandées aux salariés, en particulier quand il s'agit de travailler avec l'appui de messageries électroniques et d'ordinateurs en réseaux. Évidemment, cette référence, constante aujourd'hui, à une grande mutation du travail a quelque chose de gênant ; remarquer à quel point on fait appel à l'« intelligence » laisse entendre que ce n'était pas le cas « avant ». Ce grand partage entre taylorisme et post-taylorisme montre que dès qu'il s'agit d'évaluer la part d'abstraction dans un savoir-faire, nous pensons " écriture »/ « lecture ». C'est faire peu de cas, par exemple, de la complexité de mémorisation que demande la routinisation d'opérations de montage apparemment simples. Vincent Merle souligne que pour le montage d'une 205, les huit cent quarante variantes du modèles imposent à l'opérateur d'enregistrer un nombre colossal de combinaisons possibles dans un cycle d'une minute dix secondes. (Merle, 1993, p. 302). Autre angle d'analyse, celui de P. Zarifian, qui insiste sur l'intersubjectivité : « ... la socialisation coopératrice est en train de basculer d'une coopération réglée sur des bases fonctionnelles vers une communication intersubjective pour des raisons propres à l'efficience contemporaine du travail coopératif (...) La socialisation coopératrice reste une socialisation forcée, mais elle se spécifie aujourd'hui en requérant des bases d'accord nécessaires à la maitrise des situations industrielles que les individus doivent ensemble, identifier et gérer ». Zarifian, 1993, p. 81.

2. Pour une bibliographie, voir Lacoste, 1994 et Boutet, 1995.

3. On pourrait en dire autant d'une thèse soutenue ou d'un rapport de recherche. A propos de la relation entre la recherche et l'écriture, voir le numéro 58 de Communications, "L'écriture des sciences de l'homme ».

4. Pour Vygotsky, le langage écrit est une "fonction psychique supérieure », une "algèbre du langage ", qui "permet à l'enfant d'accéder au plan abstrait le plus élevé du langage, réorganisant par là-même aussi le système psychique antérieur du langage oral ». On peut parler de symbolisation au second degré, car le système graphique est fondé sur la mémorisation de la forme sonore. Le mot prononcé symbolise un objet du monde, le langage écrit symbolise le langage sonore. (Vygotsky, 1985, p. 260 et Schneuwly, 1988, p. 112.

5. Un produit est déclaré conforme à une norme ISO quand l'entreprise qui le fabrique et le distribue se soumet à une enquête qui rend visibles toutes les étapes du procès. Cette visibilité est obtenue par l'écriture que chaque salarié fait des actes de production ou de vérification de la qualité. Une fois rédigées, ces procédures explicitées à partir du savoir-faire "incorporé ", 
doivent être rigoureusement suivies. La fiche de procédure réalisée par chaque opérateur (décrivant le travail réel, un travail réel ou réaliste ?) devient, après validation et reformulation par les pairs ou la hiérarchie, une norme relevant du travail prescrit pour l'opérateur comme pour ceux qui seront appelés à occuper son poste. La norme ISO 9000 est coûteuse et lourde à mettre en oeuvre. Elle concerne surtout des entreprises dont les clients ou les fournisseurs font dépendre les marchés de cette norme de " qualité totale ».

6. Jacques Anis, dans le cadre plus général d'une linguistique de l'écrit, fournit des catégories qui élargissent la vision trop restrictive d'un écrit perçu comme un simple encodage de la parole ; certains éléments graphiques n'ont aucun équivalent dans la parole et interviennent dans la production du sens. Anis (1983) décompose le système graphique en trois unités : les alphagrammes, ou graphèmes alphabétiques ; les topogrammes, soit les signes de ponctuation, les blancs des mots et des paragraphes, les soulignements et topogrammes liés, c'est-à-dire ceux qui n'apparaissent qu'en surimpression des alphagrammes : la fonte du caractère ou police, son corps, son style (bas de casse, capitale, petite capitale, gras, ombré) ; les logogrammes, soit les chiffres, les sigles, les logotypes, les abréviations, les insertions iconiques.

7. Jacques Bertin a proposé une théorie de la conception graphique, la graphique, en montrant les modes de perception et de symbolisation associés à la compréhension d'un tableau de chiffres ou d'une carte ; mais ces éléments sont plutôt des aides à la conception de tableaux et à la création de graphiques qu'un appui pour analyser les produits graphiques dans les entreprises : les organigrammes, officiels ou spontanés, schématisations improvisées, croquis divers associés à la prise de notes, représentant le complexe, le procédural, la temporalité, les interconnexions entre personnes ou processus appellent des analyses discursives capable de saisir ensemble le linguistique, y compris l'abrégé ou l'acronyme, le discursif, dans ses formes asyntactiques, l'énumération, la liste, la colonne, le graphique proprement dit, l'aménagement spatial du support, le préimprimé, le formulaire, ou encore l'histogramme, le «camembert », le nuage de points, que les tableurs ont rendus courants . Voir, entre autres Bertin (1987).

8. Paru en 1830, le Journal d'un voyage à Tombouctou et à Jenné dans l'Afrique centrale, de René Caillé, connut un grand retentissement en France. Sa rédaction est en soi un exploit : les notes prises au péril de sa vie, le transport du manuscrit ébauché, les diverses réécritures en font l'objet d'une thèse sur l'écriture du voyage, de Sylvie Pavillard. Pour plus de détails, voir Parillaard (1994) et Jany (1992).

\section{RÉSUMÉS}

L'auteur fait le point sur une série d'études consacrées aux écrits de travail. Elle signale l'émergence d'une manière nouvelle d'aborder une linguistique de l'écrit qui soit une linguistique de terrain. Une telle approche peut aussi intéresser l'étude du travail. En effet l'écrit n'est pas un objet anecdotique : pour les organisations, les attentes à son égard vont au delà de la " communication », pour les salariés, l'investissement va au delà de sa fonctionnalité. C'est que l'écrit est un langage.

On mesure ici les difficultés que rencontrent ceux qui empruntent une démarche anthropologique sur de tels objets langagiers. Elles concernent aussi bien la place du chercheur dans l'entreprise que la manière dont il prendra en compte le contexte organisationnel et communicationnel : l'écrit prend sens dans un cours d'action. D'autres difficultés concernent les méthodes, dès lors qu'on veut analyser un univers qui joue de plusieurs sémiotiques. Enfin un 
effort théorique important reste à faire si l'on veut parvenir à comparer les résultats issus de terrains multiformes.

The author makes a survey of some researches making an issue of writing in the field of work. She points the emergence of a new approach to the linguistics of writing, which is part of field linguistics. Such linguistics is relevant to the study of work. Writing is neither a trivial issue from the point of view of organisations, which hope to get more from it than mere " communication ", nor from the point of view of employees, whose motivations do not remain with in the limits of the useful purposes.

Anthropological study of such linguistic stuff reads the searcher to the encountering of various difficulties. The status of the searcher in the firm where he finds the facts he studies is one of these difficulties ; so is the way he takes into account the organisational and communicational context where writings have no sense if not referred to action process. To analyse universes where various semiotic languages interfere makes the use of specific methodologies necessary. A theoretical effort remains wanted for a comparative approach of results produced in various and multiform fields.

INDEX

Keywords : professional writing, linguistics, anthropology, methodology, epistemology

Mots-clés : écriture professionnelle, linguistique, anthropologie, méthodologie, épistémologie

\section{AUTEUR}

SOPHIE PÈNE

Sophie Pène, I.U.T.Paris, A.P.L.I.S. (Analyse des Pratiques Langagières sans L'Interaction Sociale) Paris VII 